\title{
Qualidade da informação em duas empresas que utilizam Data Warehouse na perspectiva do consumidor de informação - um estudo de caso
}

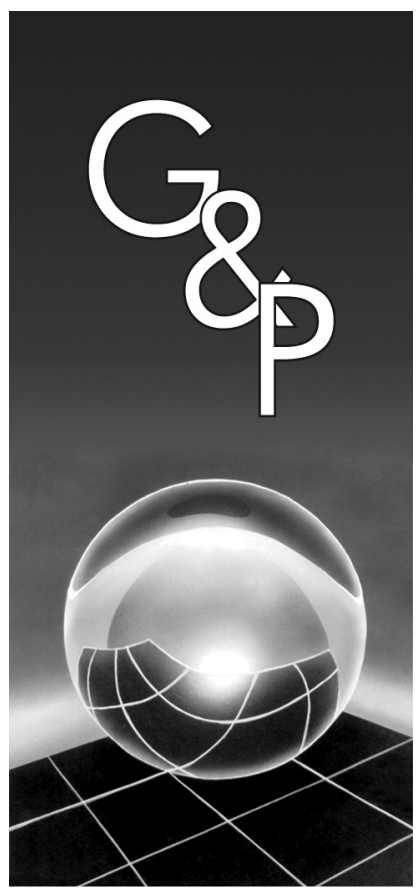

\author{
Rosana Adami Mattioda \\ Fábio Favaretto
}

\begin{abstract}
Resumo
Atualmente, convivemos com um grande volume de dados, redundantes e inconsistentes, que necessitam cada vez mais serem administrados com eficiência e transformados em informação pertinente para fins de suporte à decisão. A competitividade de uma empresa é diretamente proporcional à sua capacidade de obter informação, processála e disponibilizá-la de forma rápida, segura e com qualidade. A implantação da ferramenta Data Warehouse (DW) altera essa possibilidade para uso da informação analítica, tornando-se necessário um mecanismo de gerência da qualidade desses dados. Tendo-se em vista a necessidade de se conhecer a qualidade dessas informações, o presente estudo tem por objetivo analisar, em empresas que utilizam a ferramenta $D W$, a qualidade da informação na perspectiva do Consumidor de Informação. A análise da qualidade da informação será feita por meio de dimensões e categorias. Para maior familiaridade com o problema, com vistas a torná-lo explícito, foram feitas uma revisão da literatura e entrevistas em duas empresas, com o emprego de dois questionários, um em nível Gerencial e outro para os Consumidores de Informação. Como resultado, obteve-se a resposta de como é a qualidade da informação nestas empresas que se utilizam do DW.
\end{abstract}

Palavras-chave: Qualidade da informação. Sistema de informação. Data Warehouse. Suporte à decisão.

\section{Introdução}

No final do século XX a revolução da Tecnologia da Informação (TI) começa a convergir para a "gênese de um mundo novo". Esta revolução atua remodelando as bases materiais da sociedade e induzindo à emergência da informação como a base material de uma nova sociedade, tendo uma importância igual ou maior que a da Revolução Industrial. Uma das principais características do paradigma tecnológico informacional é que a informação é a matériaprima fundamental e não apenas informação para agir sobre a tecnologia, como foi o caso das revoluções anteriores (CASTELLS, 1999).

Nas duas últimas décadas do Século XX surgiram as primeiras iniciativas de demanda por Sistemas de Informações (SI) que pudessem suportar a tomada de decisão gerencial. À medida que o ambiente de negócios se torna mais dinâmico e competitivo, a necessidade de implementação de tais sistemas cresce significativamente, sendo necessário desenvolver sistemas para o gerenciamento da qualidade de dados, em função do volume que estes assumiram.
Enquanto as empresas lidavam com todos os recursos computacionais de processamento e gerenciamento, elas não absorviam o uso de ferramentas de apoio à decisão. Foi quando, nesse período, surgiram os conceitos Data Warehouse e de Processamento analítico On-Line ou, em inglês, On-Line Analytic Processing (OLAP), desenvolvidos a partir da necessidade das grandes corporações disporem de melhores informações para tomada de decisão, baseada em grandes volumes de dados.

A soma dos desafios tecnológicos e a própria pressão do mercado por empresas mais atentas às relações com seus clientes justificam a tendência de usar as soluções de qualidade da informação (QI) que surgiram no fim da década de 1990, as quais identificam categorias e dimensões inspiradas nos processos de gestão de qualidade da manufatura, aplicados aos SI por pesquisadores do Massachussets Institute of Technology - MIT.

O MIT, baseado no conceito referencial de que produto é igual à informação, utilizou-se dos conceitos do programa Total Quality Management - TQM, para qualidade dos 
produtos e serviços e, por analogia, montou o programa Total Data Quality Management - TDQM, dirigido pelo Prof. Richard Wang.

Segundo Burgess, Gray e Fiddian (2004), muitos trabalhos atualmente conduzidos na área de pesquisa da QI focaram a qualidade a partir da perspectiva organizacional ou da produção de informação. A perspectiva de qualidade do Consumidor de Informação (CI) difere desta, sendo que este não tem controle sobre a QI disponível e seu objetivo é encontrar informação que atenda a suas necessidades pessoais, ao invés de prover informação que atenda à necessidade de outros.

Levando-se em consideração que as questões acima relatadas lidam com ligações operacionais que necessitam ser traçadas ao longo do tempo, em vez de serem encaradas como mera repetição ou incidência, a presente pesquisa irá descrever e analisar a seguinte questão: como é a Qualidade da Informação gerada pelo Data Warehouse na perspectiva do Consumidor de Informação?

O principal objetivo deste estudo de caso é analisar a QI em duas empresas que utilizam o DW na perspectiva do CI e verificar o grau de importância atribuída às dimensões e categorias da qualidade da informação. O sujeito do estudo é o Consumidor de Informação, por utilizar o produto de informação na sua rotina de trabalho e estar diretamente associado à utilização desses dados.

Este artigo é iniciado com esta introdução, seguida da revisão bibliográfica dos conceitos empregados. A seguir, é apresentada descrição da metodologia utilizada. É apresentado então o estudo de caso realizado, começando pela sua preparação e passando pelo seu desenvolvimento. Finalizando, são apresentadas as conclusões obtidas.

\section{Revisão bibliográfica}

\subsection{Estágio de informatização}

Segundo Falsarella e Chaves (1995), uma grande quantidade de termos é usada para classificar e caracterizar SI. Uma forma de limitar e demarcar corretamente as fronteiras conceituais entre esses termos é relacionar cada tipo de SI com o estágio de utilização da informática em que uma organização se encontra. O uso da TI dentro das organizações tende a seguir um padrão previsível no tempo, como descreve o autor Richard Nolan apud Falsarella e Chaves (1995), em seu livro Management Accounting and Control of Data Processing, que foi o primeiro a analisar estes padrões em relação à adoção de novas tecnologias de processamento de informação.

De acordo com o modelo de Nolan (1977), a informática evolui segundo uma sequência definida de estágios, os quais representam a familiaridade e o domínio crescentes da tecnologia pela empresa. Cada estágio é caracterizado por um tipo de aplicação preponderante, forma de planejamento e controle da informática e tecnologia empregada. Abaixo, a Tabela 1 sintetiza os estágios da informatização descritos por Nolan e sua respectiva forma, caracterizada por Falsarella e Chaves (1995).

\subsection{Data Warehouse}

Inmon (1997) foi o primeiro a cunhar o termo Data Warehouse (ou armazém de dados) para repositórios analíticos corporativos, e sua definição clássica é a de "uma coleção de dados orientada por assunto, integrada, variante e não volátil, que tem por objetivo dar suporte aos processos de tomada de decisão". A Figura 1 detalha o processo descrito pelo autor.

O termo Data Warehouse, segundo Singh (2001), é utilizado para definir

um conjunto de novos conceitos e ferramentas que evolui para uma tecnologia que permite atacar o problema de oferecer a todas as pessoas-chave da empresa acesso a qualquer nível de informação necessário para que a organização possa sobreviver e prosperar em um mundo cada vez mais competitivo Singh (2001).

Um DW é um lugar em que os dados ficam armazenados de forma que as aplicações possam acessá-los e compartilhá-los facilmente. Armazena dados no formato read-only (somente leitura) e coleta informações de várias fontes diferentes, sendo utilizado como local em que essas diferenças se tornam compatíveis, e é colocado em um warehouse (armazém) para que vários aplicativos usem as mesmas informações.

Ainda para o autor o subconjunto do DW é o Data Mart que "desempenha o papel de um DW (departamental, regional ou funcional), podendo-se construir uma série deles ao longo do tempo e eventualmente vinculá-los através de um DW lógico empresa-inteira".

\subsection{Produto de informação e Total Data Quality Management}

Conforme Wang, Ziad e Lee (2000), a TDQM, analogamente ao ciclo PDCA (Planejar-Plan, Fazer-Do, Verificar-Check, Agir-Act), compreende quatro passos (Figura 2). O primeiro passo é definir os requisitos de qualidade e as métricas a serem adotadas. Em seguida, deve-se medir a qualidade ao longo do ciclo de vida da informação nos sistemas e processos que a produzem. $\mathrm{O}$ terceiro passo, é a analise e identificação de potenciais causas dos problemas de qualidade. Por fim, especificam-se e implementam-se as melhorias nos processos e sistemas para manter a qualidade dos dados e prevenir eventuais vulnerabilidades.

Para Wang (1998), o propósito da metodologia TDQM é a entrega de produtos de informação (PI) de alta qualidade para os CI. Visa facilitar a implementação em uma organização como um todo de uma política de qualidade de dados 
Tabela 1. Estágios de informatização e forma caracterizada.

\begin{tabular}{|c|c|}
\hline Estágios da informatização - Nolan (1977) & Forma caracterizada - Falsarella/Chaves (1995) \\
\hline $\begin{array}{l}\text { Iniciação: Neste estágio, o usuário é resistente ao uso da } \\
\text { informática e seu envolvimento com a tecnologia é superficial. A } \\
\text { organização encoraja o uso da informática e se preocupa com o } \\
\text { aprendizado, mas poucas atividades são automatizadas. } \\
\text { Contágio: Neste estágio, começam a proliferar SI informatizados, } \\
\text { que automatizam atividades antes desenvolvidas manualmente, } \\
\text { sem, porém, se preocupar com a integração das informações. }\end{array}$ & $\begin{array}{l}\text { Sistemas Transacionais: Também identificados pela expressão } \\
\text { Electronic Data Processing (EDP), são necessários para o controle } \\
\text { operacional das organizações. } \\
\text { No modelo da evolução da informática nas organizações proposto } \\
\text { por Richard Nolan, os SI transacionais se enquadram nos estágios } \\
\text { de iniciação e contágio. }\end{array}$ \\
\hline $\begin{array}{l}\text { Controle: Neste estágio, o crescimento do uso de SI na } \\
\text { organização passa a ser explosivo, sendo o usuário a força } \\
\text { propulsora. Por isso, a organização passa a exigir melhor gestão } \\
\text { dos recursos de informática. } \\
\text { Integração: Neste estágio, em resposta à pressão por melhor } \\
\text { gestão, os SI passam a ser orientados para atender às necessidades } \\
\text { dos níveis gerenciais, as informações são de melhor qualidade e é } \\
\text { exigida maior integração entre elas. }\end{array}$ & $\begin{array}{l}\text { Sistemas Gerenciais: Proveem material para análise, } \\
\text { planejamento e suporte à decisão. } \\
\text { São geralmente chamados de Management Information Systems } \\
\text { (MIS). O surgimento desses sistemas acontece nos estágios de } \\
\text { controle e integração no modelo proposto por Richard Nolan, em } \\
\text { que o usuário é força propulsora e exige informações em maior } \\
\text { quantidade, menor tempo e com melhor nível de integração. }\end{array}$ \\
\hline $\begin{array}{l}\text { Administração de Dados: Neste estágio, os SI começam a ser } \\
\text { organizados em termos de sistemas que interessam à organização } \\
\text { como um todo (chamados corporativos) e sistemas de uso setorial } \\
\text { ou especializado, havendo cuidado, em qualquer hipótese, com a } \\
\text { correta administração dos dados, de modo a evitar redundâncias. } \\
\text { Maturidade: Neste estágio, a informação passa a ser considerada } \\
\text { como patrimônio da organização, o usuário é participativo e } \\
\text { responsável e o crescimento da informática é ordenado. }\end{array}$ & $\begin{array}{l}\text { Sistemas Executivos: Com base nos dados existentes nos EDP, } \\
\text { nas informações disponíveis nos MIS e em informações coletadas } \\
\text { de fontes externas à organização, é possível construir SI dirigidos } \\
\text { para a alta gerência. São geralmente chamados de Executive } \\
\text { Information Systems (EIS). } \\
\text { No modelo definido por Richard Nolan, os EIS começam a ser } \\
\text { desenvolvidos nas organizações nos estágios de administração } \\
\text { de dados e maturidade. Nesses estágios, os SI existentes refletem } \\
\text { o fluxo de informações da organização, o usuário participa } \\
\text { integralmente do desenvolvimento dos sistemas, as informações } \\
\text { passam a ser consideradas patrimônio da organização, o } \\
\text { crescimento da informática é ordenado, a informática passa a ter } \\
\text { função de apoio estratégico para a organização e não se tomam } \\
\text { decisões sem base nas informações produzidas por um EIS. }\end{array}$ \\
\hline
\end{tabular}

Fonte: Adaptado de Falsarella e Chaves (1995).

formalmente expressa como gerenciamento de alto nível. Fundamental para esta metodologia é a premissa de que as organizações devem tratar a informação como um produto que se move através dos "sistemas de manufaturas de informações", de forma muito parecida com a de um produto físico.

A seguir, a Tabela 2 descreve uma analogia entre manufatura de produto e manufatura de informação, criada para a prática de qualidade de dados.

Com a definição de um processo de produção da informação, é possível também fazer uma analogia da aplicação das ferramentas e abordagens de qualidade aplicadas a produtos, existindo assim a oportunidade de se trabalhar de forma objetiva com a qualidade da informação (FAVARETTO; MATTIODA, 2005).

Uma analogia de aplicação de ferramenta de qualidade pode ser feita sobre o conceito de melhoramento contínuo, que implica literalmente um processo sem fim, questionando repetidamente e requestionando os trabalhos detalhados de uma operação do TQM. A natureza repetida e cíclica
Tabela 2. Analogia manufatura de produto versus manufatura de informação.

\begin{tabular}{ccc}
\hline $\begin{array}{c}\text { Sistema de } \\
\text { manufatura }\end{array}$ & $\begin{array}{c}\text { Manufatura do } \\
\text { produto }\end{array}$ & $\begin{array}{c}\text { Manufatura da } \\
\text { informação }\end{array}$ \\
\hline Entrada & Material bruto & Dados brutos \\
Processo & Linha de montagem & $\begin{array}{c}\text { Sistema de informação } \\
\text { Saída }\end{array}$ \\
\hline
\end{tabular}

Fonte: Wang, Ziad e Lee (2000).

do melhoramento contínuo é mais bem resumida do que é o chamado ciclo PDCA.

Conforme Wang, Ziad e Lee (2000), a TDQM, fazendo uma analogia ao ciclo PDCA, compreende quatro passos:

1. Definir os requisitos de qualidade e as métricas a serem adotadas;

2. Medir a qualidade ao longo do ciclo de vida da informação nos sistemas e processos que a produzem; 


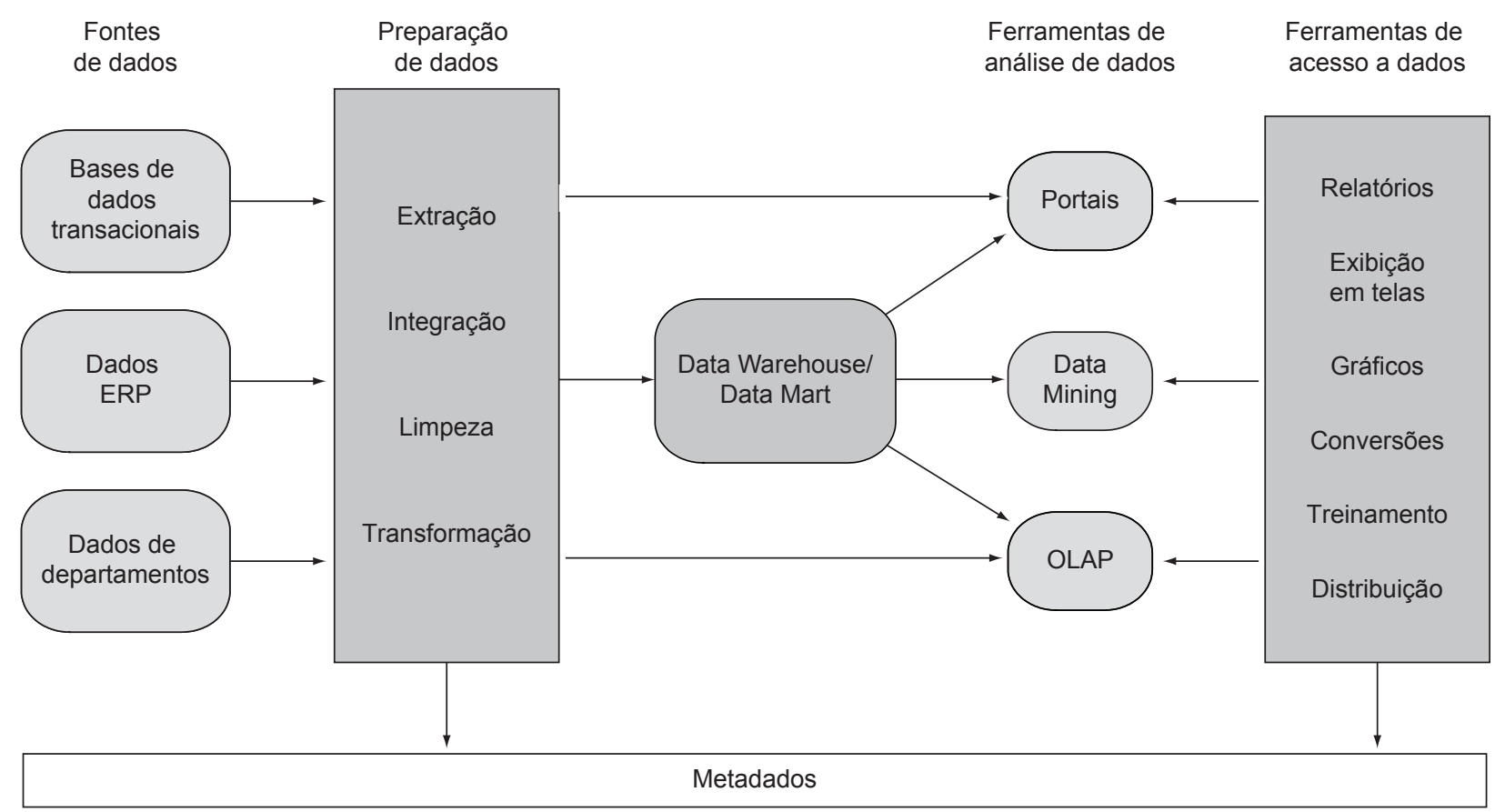

Figura 1. Processo do Data Warehouse. Fonte: Adaptado de Inmon (1997).

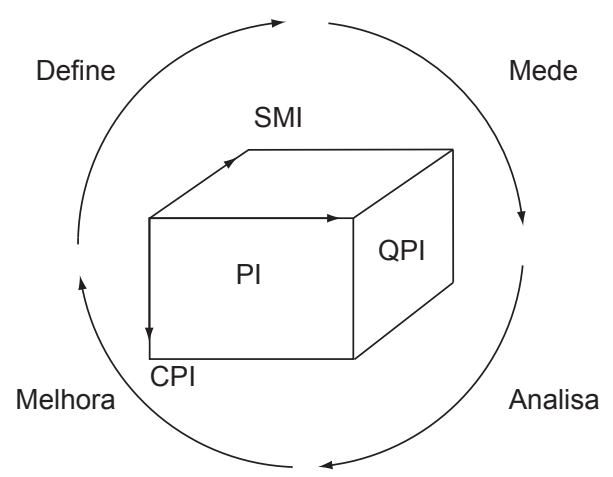

$\mathrm{PI}$ (Produto de Informação)

CPI (Características PI)

QPI (Qualidade PI)

SMI (Sistema de Manufatura de Informações)

Figura 2. Ciclo PDCA para o TDQM. Fonte:Adaptado de Wang, Ziad e Lee (2000).

3. Analisar e identificar potenciais causas dos problemas de qualidade; e

4. Especificar e implementar as melhorias nos processos e sistemas para manter a qualidade dos dados e prevenir eventuais vulnerabilidades.

\subsection{Dimensões da qualidade da informação}

Para Al-Hakim (2004), assim como no gerenciamento de produtos físicos, a QI tem múltiplas dimensões. A escolha destas dimensões foi primeiramente baseada no entendimento intuitivo, experiência individual ou revisão bibliográfica e depende do uso atual da informação.

Segundo Burgess, Gray e Fiddian (2004), a despeito de todas as pesquisas conduzidas na tentativa de criar uma definição de qualidade "não existe uma definição padrão única para qualidade". A maioria também concorda que os múltiplos atributos usados para definir qualidade podem ser agrupados em categorias relacionadas, representando uma estrutura hierárquica.

Na Tabela 3, encontram-se as Categorias, Dimensões e Definições, adaptadas de alguns autores, focando principalmente as definições das dimensões da qualidade.

Um problema provocado pela má qualidade de dados, muito pior que o custo direto, é o descrédito interno e externo e suas consequências sobre os processos de tomada de decisão e sobre a percepção que os clientes e fornecedores formam acerca dos sistemas da empresa. Por outro lado, a qualidade de dados da organização pode ser considerada uma vantagem competitiva. Quando isso acontece, é possível identificar rapidamente potenciais oportunidades de negócio ou de marketing a partir de transformações e de análises sobre os dados comercias, atuais e históricos. Ainda são poucas as organizações que adotam práticas estruturadas de gestão de dados e, sem esta prática, é certa a exposição a riscos e falhas nas iniciativas de Tecnologia de Informação e de negócio. (MATTIODA; FAVARETO, 2006).

Para Strong, Lee e Wang (1997a), uma qualidade pobre de informação pode gerar o caos, a menos que a causa seja 
Tabela 3. Categorias, dimensões e definições da QI.

\begin{tabular}{|c|c|c|}
\hline Categoria & Dimensão & Definição \\
\hline \multirow[t]{4}{*}{ Intrínseca } & Acuracidade (accuracy) & Quanto a informação é correta e confiável. \\
\hline & Credibilidade (believability) & Quanto a informação é considerada como verdadeira e verossímil. \\
\hline & Objetividade (objectivity) & Quanto a informação é imparcial. \\
\hline & $\begin{array}{l}\text { Reputação } \\
\text { (reputation) }\end{array}$ & $\begin{array}{l}\text { Quanto a informação é altamente considerada } \\
\text { em termos de sua fonte ou conteúdo. }\end{array}$ \\
\hline \multirow[t]{2}{*}{ Acessibilidade } & $\begin{array}{l}\text { Acessibilidade } \\
\text { (accessibility) }\end{array}$ & $\begin{array}{l}\text { Quanto a informação está } \\
\text { disponível, ou fácil e rapidamente recuperável. }\end{array}$ \\
\hline & $\begin{array}{l}\text { Segurança no acesso } \\
\text { (Access security) }\end{array}$ & $\begin{array}{l}\text { Quanto o acesso à informação } \\
\text { é restrito apropriadamente para manter sua segurança. }\end{array}$ \\
\hline \multirow[t]{5}{*}{ Contextual } & $\begin{array}{l}\text { Integridade/Completeza/Perfeição } \\
\text { (Completeness) }\end{array}$ & $\begin{array}{l}\text { Quanto a informação não está } \\
\text { extraviada e é suficiente para a tarefa em amplitude e profundidade. }\end{array}$ \\
\hline & $\begin{array}{l}\text { Quantidade de informação apropriada } \\
\text { (appropriate amount) }\end{array}$ & $\begin{array}{l}\text { Quanto o volume da informação é } \\
\text { apropriado para a tarefa ser executada. }\end{array}$ \\
\hline & Relevância (relevancy) & Quanto a informação é aplicável e útil para a tarefa a ser realizada. \\
\hline & $\begin{array}{l}\text { Temporalidade/Oportunidade } \\
\text { (timeliness) }\end{array}$ & $\begin{array}{l}\text { Quanto a informação está suficientemente } \\
\text { atualizada para a tarefa a ser realizada. }\end{array}$ \\
\hline & Valor Agregado (Value-added) & Quanto a informação é benéfica e proporciona vantagens por seu uso. \\
\hline \multirow[t]{5}{*}{ Representativa } & $\begin{array}{l}\text { Facilidade de entendimento } \\
\text { (ease of understanding ) }\end{array}$ & Quanto a informação é facilmente compreendida. \\
\hline & $\begin{array}{l}\text { Facilidade de manipulação/operação } \\
\text { (ease of manipulation/operacion) }\end{array}$ & $\begin{array}{l}\text { Quanto a informação é } \\
\text { fácil de ser manipulada e aplicada em diferentes tarefas. }\end{array}$ \\
\hline & $\begin{array}{l}\text { Interpretabilidade } \\
\text { (interpretability) }\end{array}$ & $\begin{array}{l}\text { Quanto a informação está em linguagem apropriada, } \\
\text { símbolos e unidades, e as definições são claras. }\end{array}$ \\
\hline & $\begin{array}{l}\text { Representação concisa } \\
\text { (concise representation) }\end{array}$ & Quanto a informação está compactamente representada. \\
\hline & $\begin{array}{l}\text { Representação consistente } \\
\text { (consistent representation) }\end{array}$ & Quanto a informação é apresentada em um mesmo formato. \\
\hline
\end{tabular}

Fonte: Adaptado de Wang; Ziad e Lee (2000), Strong e Wang (2002), Lee et al. (2002) e Pipino, Lee e Wang (2002).

diagnosticada. Esforços para sua melhoria seriam similares a tentar remendar buracos de uma estrada. Os autores descrevem as dez causas-chave, sinais de alerta e remendos típicos, e seus respectivos processos e efeitos na organização, considerando também as dimensões afetadas.

\subsection{A Informação na perspectiva do consumidor de informação}

Conforme Arndt e Langbein (2002), qualidade de dados é o "encontro consistente das expectativas dos consumidores de informação".

Para o consumidor de dados, um dado pronto para uso significa que: é acurado, é crível, é objetivo, é relevante, é oportuno, tem reputação, tem valor agregado, é apropriado em quantidade, tem representação concisa consistente e completa, é interpretável, é acessível, compreensível e seguro (WANG; STRONG, 1996).

O papel de um SI é proporcionar uma representação de um domínio de aplicação (também chamado sistema de mundo real) conforme percebido pelos usuários e, portanto, as visões dos usuários servem de padrão contra o que é definido como qualidade de dados (WAND; WANG, 1996).

Essa diferença de foco significa que as definições de qualidade que têm surgido para uso por provedores de informação não são apropriadas para o CI. O típico CI quer encontrar a melhor informação disponível que atenda a seus requisitos, num momento apropriado e no seu domínio de interesse atual. Isso pode não necessariamente ser o melhor resultado possível, na medida em que o consumidor 
sempre tem restrições, tal como o tempo disponível para pesquisar a informação.

Uma classificação diferente da dimensão da qualidade de dados foi desenvolvida por Angeles (2004) baseada na perspectiva do consumidor de dados. Categorias de qualidade de dados foram identificadas como intrínseca, acessibilidade, contextual e representativa, sendo o método de medida da qualidade de dados não endereçado. Sobre papéis desempenhados na manufatura de informações, Wang, Ziad e Lee (2000) definem três papéis:

1. Coletores de Informações: criam, coletam ou abastecem/ suprem os dados para o produto de informação. Estão associados ao processo de produção de dados;

2. Guardiões de informações: projetam, desenvolvem ou conservam a infraestrutura de sistema e dados para o produto de informação. Estão associados ao armazenamento, manutenção e segurança; e

3. Consumidores de Informações: utilizam o produto de informação no seu trabalho e estão associados ao processo de utilização dos dados, podendo implicar agregação de dados adicionais e integração.

\section{Metodologia aplicada}

Tendo em vista que a abordagem principal do problema é a avaliação da qualidade da informação gerada pela ferramenta DW em duas organizações, o estudo terá como instrumento principal a pesquisa qualitativa descritiva, assumindo a forma de estudo de caso.

Sendo o objetivo a condução de uma pesquisa em que o pesquisador é investigador-observador não participante e sendo o Data Warehouse um Sistema de Informação, o estudo de caso é a metodologia apontada como particularmente apropriada para problemas como este em que a pesquisa e teoria estão em estágio inicial de formação. $O$ foco não é o DW, mas o comportamento dos CI em face da utilização da ferramenta. A unidade de análise do projeto de pesquisa é a qualidade de informação.

A Tabela 4 descreve as respostas que determinaram que o estudo de caso é a metodologia mais adequada para aplicação do presente estudo.

Para esta pesquisa foram elaborados dois questionários, um gerencial e outro para os CI, sendo a coleta de informações realizada por meio de entrevistas pessoais. $\mathrm{O}$ instrumento de pesquisa foi estruturado e formatado por meio de um roteiro/protocolo. Neste artigo serão analisadas somente as questões referentes aos CI.

Como característica principal para a coleta de dados, tem-se a utilização da ferramenta DW e de elementos comuns com os objetivos propostos. As etapas para a seleção da amostra e aplicação da pesquisa foram as seguintes:

1. Identificação das empresas que tinham implementada a ferramenta DW e levantamento das seguintes variáveis, como critérios: estágio de informatização, tempo de implementação (no mínimo dois anos) e número de usuários;

2. Visita a no mínimo uma empresa, para o levantamento e formulação dos itens a serem avaliados e coleta de informações de fontes secundárias;

3. Definição do número de empresas a serem pesquisadas (número de casos);

4. Confecção do instrumento de pesquisa,

5. Aplicação do pré-teste do questionário para identificar os elementos dos objetivos propostos na abrangência metodológica e correção do questionário;

6. Montagem de um banco de dados;

7. Aplicação da entrevista com o maior número possível de CI;

8. Digitação dos dados no banco de dados;

9. Análise e comparação das informações; e

10. Redação do relatório final.

Para a realização do estudo, encontrou-se dificuldade para obtenção do cadastro de empresas que utilizassem a ferramenta DW, assim como qualquer outro dado quanto à utilização de SI.

Como forma de contornar tal problema e levantar dados quanto ao uso da tecnologia DW pelas empresas, foi feito contato com um representante regional de um desenvolvedor de software atuante em nível mundial e indagou-se, inicialmente, sobre o perfil das empresas na Cidade de Curitiba e Região Metropolitana que utilizavam a ferramenta. No perfil dessas empresas apresentadas pelo representante regional da desenvolvedora mundial de software e posterior revisão literária, verificaram-se como características de maior ocorrência:

1. Serem empresas com o estágio do Sistema de Informação compreendido, no estágio de classificação de Nolan (1977), entre Integração, Administração de Dados e Maturidade e caracterizadas por Falsarella e Chaves (1995) entre Sistemas Gerenciais, Executivos, Especialistas e de Apoio à Decisão.

2. Serem empresas que já tinham um histórico de implementação e estivessem utilizando sistematicamente a ferramenta. Neste caso em particular, cumpre ressaltar que foi informado pelo representante da desenvolvedora mundial de software que, para a utilização sistemática da ferramenta, o tempo necessário para a implementação do DW seria de, no mínimo, dois anos.

Uma vez estabelecidas as características, estágio de classificação de SI e tempo de implementação, solicitou-se ao representante da empresa mundial de software a possibilidade de indicação nominal das empresas que atendessem tais requisitos, respectivos contatos e que, também, se pudesse ter acesso as elas para a aplicação do presente estudo. 
Tabela 4. Respostas às questões para a escolha do estudo de caso.

\begin{tabular}{ll}
\hline \multicolumn{1}{c}{ Pergunta } & \multicolumn{1}{c}{ Resposta para a aplicação no presente estudo } \\
\hline O fenômeno de interesse pode ser estudado fora de seu ambiente natural? & $\begin{array}{l}\text { Não. O objetivo é verificar a Qualidade da Informação com } \\
\text { utilização do DW. }\end{array}$ \\
O estudo focaliza eventos contemporâneos? & $\begin{array}{l}\text { Sim. O objetivo é observar o comportamento dos CI, com a } \\
\text { tecnologia atualmente disponível. }\end{array}$ \\
O controle ou a manipulação dos sujeitos ou eventos é necessário? & $\begin{array}{l}\text { Não. O objetivo é identificar elementos técnicos e } \\
\text { comportamentais. } \\
\text { O fenômeno de interesse tem uma base teórica estabelecida? }\end{array}$ \\
& $\begin{array}{l}\text { Navaliação da QI com a utilização do DW não é } \\
\text { frequentemente encontrada na literatura. }\end{array}$ \\
\hline
\end{tabular}

Fonte: Adaptado de Pozzebon e Freitas (1997).

Sete empresas foram relacionadas. Feitos os contatos iniciais, apenas uma demonstrou interesse. As outras alegaram estar em fase de mudança, não ter interesse ou, simplesmente, desconhecer a existência da ferramenta na empresa e mesmo o nome/conceito da ferramenta. Junto à empresa contatada e interessada, foi feita inicialmente uma reunião com o responsável pela implantação do DW, na qual foram apresentadas as ideias do projeto e, também, levantadas informações sobre o ambiente de estudo e possíveis questionamentos.

Em passo posterior, decidiu-se que, para a aplicação do presente estudo, seria realizada uma pesquisa na empresa já contatada e que havia manifestado interesse e, também, em uma segunda empresa posteriormente indicada e que manifestou interesse em participar do presente estudo.

A seguir, apresentam-se informações sobre as empresas pesquisadas, ambas instaladas no Município de Curitiba - PR.

A empresa A é um órgão da Administração Pública Estadual, atuante há mais de 50 anos no Estado do Paraná. Visando agilizar o processo de obtenção de informações, preocupa-se com a modernização de seus sistemas, que passaram a subsidiar as análises em âmbito Estadual.

As decisões que levaram à compra e adoção da ferramenta DW foram: a facilidade de uso, interface em português e o treinamento em Curitiba. Para o desenvolvimento do projeto de implantação, a solução encontrada pela referida empresa foi o desenvolvimento interno, com apoio de consultoria externa. Seguiram-se as seguintes etapas para o desenvolvimento do projeto de implementação: aprendizado sobre a ferramenta, levantamento das necessidades, desenvolvimento, treinamento dos usuários e sucessivos aprimoramentos.

O tempo de implantação da ferramenta DW na Empresa A é de aproximadamente cinco anos e o responsável pela implantação do DW classifica o tipo de sistema atualmente utilizado pela empresa de Especialista. Este sistema é conhecido como Expert Systems (ES), quando fornece, ele mesmo, soluções para determinados problemas, e como Expert Support Systems (ESS), quando fornece informações extraídas das bases de conhecimento a profissionais e executivos, para auxiliá-los no processo de tomada de decisão.

Com base nessas informações, conclui-se que a Empresa A encontra-se no estágio de Integração. Neste estágio, que ocorre em resposta à pressão por melhor gestão e é orientado para atender às necessidades dos níveis gerenciais, as informações são de melhor qualidade e é exigida maior integração entre elas.

A empresa B é uma empresa estrangeira do setor industrial, que inaugurou sua fábrica em Curitiba há quase trinta anos. Seu produto é líder absoluto em tecnologia agregada e vendas no mercado brasileiro. Desde a implantação da fábrica no Brasil, preocupou-se com a qualidade de seus produtos e serviços, sendo uma das pioneiras na implantação de Sistemas de Gestão da Qualidade. Atualmente exporta seus produtos para aproximadamente 30 países.

Segundo a gerência, a decisão que levou à compra e adoção da ferramenta foi a melhoria no tratamento das informações gerenciais, tendo como dificuldade na implantação a determinação da carga de dados históricos, sendo que, atualmente, nessa filial, só está implementada no setor de Vendas.

A utilização do DW no fluxo do processo decisório ocorre: na avaliação do desempenho da área de vendas, em campanhas promocionais e definição de visitas que são realizadas baseadas nas informações geradas.

O tempo de implantação da ferramenta DW na Empresa B é de aproximadamente dois anos e a gerência responsável pela implantação do DW classifica o tipo de sistema atualmente utilizado pela empresa de Especialista e Gerencial. O Sistema Especialista, como já descrito, é conhecido como Expert Systems (ES) ou como Expert Support Systems (ESS). O Sistema Gerencial provê material para análise, planejamento e suporte à decisão, podendo ser chamado também de Management Information Systems (MIS). Com essas informações, conclui-se que a Empresa B encontra-se no estágio de Integração, em que o usuário é a força propulsora e exige informações em maior quantidade, menor tempo e com melhor nível de integração. Suas informações auxiliam a Gerência no processo decisório. 


\section{Preparação do estudo de caso}

Como forma de possibilitar a verificação e análise do quão importante na perspectiva do Consumidor de Informação é a Qualidade da Informação gerada pelo DW, o presente estudo foi conduzido seguindo uma metodologia de três fases sequenciais, conforme indicadas na Figura 3.

Como princípio básico e para que os entrevistados fossem considerados parte do estudo, ou seja, consumidores de informação (CI), ficou estabelecido que: tivessem que ter os dados acessíveis; deveriam ser capazes de interpretar os dados; os dados deveriam ser relevantes; e eles deveriam encontrar os dados exatos.

\section{Estudo de caso}

A seguir serão apresentadas as considerações relativas à análise do presente estudo de caso, na percepção do Consumidor de Informação, de acordo com as etapas de Análise do Estudo de Caso. Para cada fase, foram analisados itens, conforme apresentado na Figura 3. Esta análise é baseada nas respostas aos questionários, nos quais foram aplicadas perguntas objetivas relacionadas ao item analisado e com respostas em uma escala de concordância de 5 itens (de "não concordo" até "concordo plenamente").

\subsection{Fase I - fatores limitantes}

Existem variáveis que estão relacionadas à aplicação do presente estudo. Por suposição, essas variáveis são limitantes do estudo, uma vez que, sem um tempo hábil de implementação e consequentemente seu estágio de informatização, não existiria a familiaridade e o domínio da ferramenta. Os itens analisados nesta fase são:

Tempo de implementação - Foi destacado por Sakaguchi e Frolick (1997), que colocam como uma desvantagem as empresas não terem o tempo necessário para a conclusão de todo o projeto, quer seja pela complexidade e alto custo de desenvolvimento, ou pela administração e treinamento. Neste contexto, em uma coleta de dados auxiliar feita com o representante regional da desenvolvedora mundial de software contatado, este atribuiu um tempo mínimo para a implementação do DW de dois anos. O DW foi implementado há cinco anos na Empresa A e há aproximadamente dois anos na Empresa B. A Empresa B é considerada no presente estudo como tendo um Sistema de Informação Coorporativo, em que a ferramenta encontra-se implementada dentro do processo, apenas no setor de Vendas.

Estágio de informatização - Conforme o estudo de Nolan (1977), a informática evolui segundo uma sequência definida de estágios, os quais representam a familiaridade e o domínio crescentes da tecnologia pela empresa. O tipo de Sistema atualmente utilizado pelas empresas A e B é o Sistema Especialista, sendo que a Empresa B também o denominou como Sistema Gerencial. Ambas as empresas se encontram no estágio de Integração que, segundo a classificação de Nolan, neste estágio, em resposta à pressão por melhor gestão, os SI passam a ser orientados para atender às necessidades dos níveis gerenciais, nos quais as informações são de melhor qualidade, sendo exigida maior integração entre elas.

Motivação para o uso do DW - O sucesso da implementação da ferramenta DW e sua continuidade dependem, principalmente, da motivação para que as pessoas responsáveis pela tomada de decisão o utilizem. Conforme Doherty e Doig (2003), o sucesso de um Sistema de Apoio à Decisão (SAD), a sua continuidade e, principalmente, a motivação para que as pessoas responsáveis pela tomada de decisão o utilizem, dependem dos seguintes fatores:

1. O modelo construído deve atender às necessidades gerais da organização e não somente às necessidades específicas de um usuário;

2. Eventuais mudanças no sistema devem ser feitas rapidamente pelo analista de sistemas para atender a novas necessidades de informação para apoio à decisão;

3. Informações sobre as decisões tomadas devem ser armazenadas e estarem disponíveis para que outras pessoas as utilizem em novos processos de tomada de decisão;

4. A interface com o usuário deve ser a mais amigável possível;

5. A obtenção das informações, internas e externas à organização, deve ser imediata;

6. Os benefícios da utilização de SAD devem ser disseminados na organização por meio de cursos, palestras, etc.

Na Empresa A, os CI “concordam em parte" que existe motivação para o uso da ferramenta DW, sendo os seguintes itens melhor avaliados: o modelo atende às necessidades da organização, as mudanças que são feitas no sistema e a interface com o usuário devem ser fáceis e amigáveis.

$\mathrm{Na}$ Empresa B, os CI "discordam em parte" quanto à motivação para o uso da ferramenta. Nas melhores avaliações, tem-se: a obtenção de informações externas / internas serem imediatas e a interface com o usuário ser fácil e amigável. Esse resultado era esperado no caso dessa empresa B, uma vez que o DW está implementado somente no setor de vendas há dois anos e, nos outros setores, ainda está em início de implantação.

Tanto na Empresa A quanto na empresa B, não existe gerenciamento específico das informações, e o tempo de implantação da ferramenta é diferente, porém, verifica-se que, independentemente destes aspectos, existe a motivação 
Análise de estudo de caso

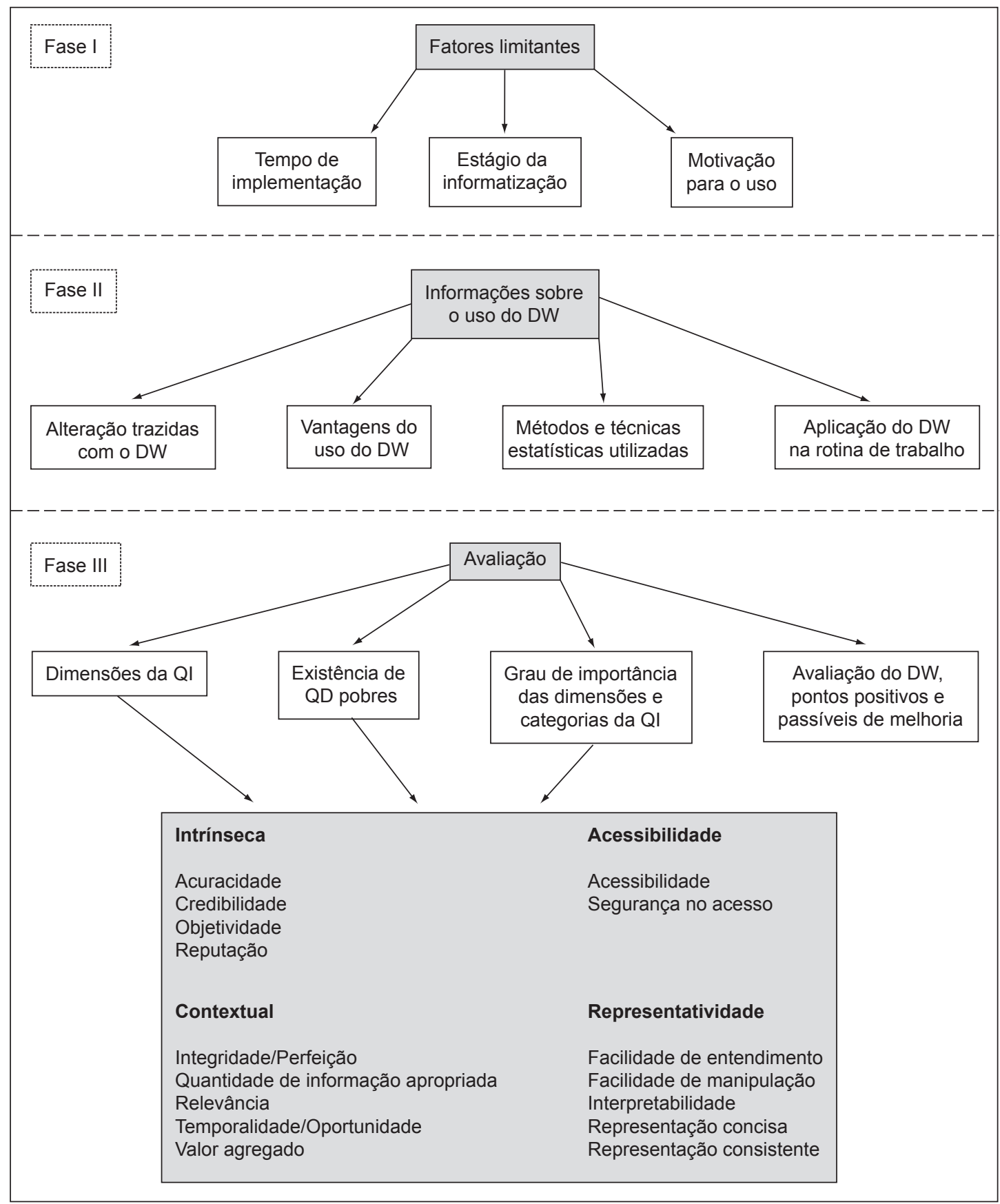

Figura 3. Etapas da análise do estudo de caso.

para o uso do DW no que diz respeito a este possuir uma interface com o usuário fácil e amigável.

\subsection{Fase II - informações sobre o uso do Data Warehouse}

As informações sobre o uso do DW mostram a utilização da ferramenta, o envolvimento e entendimento do entrevistado e, também, o ambiente. Analogamente a um organismo vivo, o processo de Data Warehousing não se encerra, e o DW corporativo certamente continuará a evoluir adequando-se às novas demandas de informação analíticas que surgirão em função de mudanças na organização e no ambiente de negócios. No levantamento sobre o uso do DW, os seguintes itens foram analisados:

Vantagens da utilização do DW - Os itens utilizados foram extraídos do estudo feito por Sakaguchi e 
Frolick (1997) sobre vários casos de empresas que já estavam utilizando ou estavam no processo inicial de construção de um DW. A intenção é conhecer, na percepção do CI, quais vantagens são consideradas mais importantes.

Nas duas empresas, os CI "concordam em parte" com as vantagens do uso do DW. Na percepção dos CI dessas empresas, as maiores vantagens da utilização da ferramenta DW estão na infraestrutura computacional e na segurança. Outra vantagem apontada, esta pela Empresa B, foi o custo da operação. Este item é interessante, pois como se trata de uma organização coorporativa, existe preocupação quanto a custos.

Na Empresa A, os CI "não concordam nem discordam" das vantagens do uso da ferramenta no que diz respeito à simplicidade e habilitação para o processamento paralelo aos SI transacionais. Já na Empresa B, os CI “discordam em parte" também da simplicidade e da administração do fluxo da informação. Para as duas Empresas, a vantagem em relação à simplicidade foi o item menos percebido pelos CI.

Métodos e técnicas estatísticas utilizadas - Captura o entendimento que o CI tem sobre o processo de extração de conhecimento com a aplicação da estatística. Importante frisar-se que pela revisão literária sabese, a priori, que estes modelos são frequentemente subutilizados, sendo que os CI preferem métodos que, para guiar suas decisões e ações, exijam menos esforço ou preparo para processar as informações em seus ambientes. Para Chenoweth, Dowling e St. Louis (2004), o grande desafio está em aumentar a consciência da necessidade da informação melhorada obtida por modelos complexos.

Nesta questão, entendeu-se que havia desconhecimento conceitual por parte dos entrevistados. Não sendo este fato inicialmente identificado, consequentemente não existiu na conscientização inicial uma explicação sobre o assunto antes da aplicação do questionário. Assim sendo, foi considerado que as respostas estão com vieses de interpretação. Porém, é importante mostrar que um dos desafios está em aumentar a consciência da necessidade da informação melhorada obtida por modelos complexos. Entende-se que deve o aprendizado acadêmico se adequar a essas mudanças, uma vez que todas as ferramentas estatísticas podem ser aplicadas e servem para equacionar qualquer fenômeno, principalmente no processo decisório.

Alterações trazidas com a opção de trabalhar com o DW e aplicação do DW na rotina de trabalho

$\mathrm{Na}$ Empresa A, a grande maioria utiliza diariamente a ferramenta DW em sua rotina de trabalho, principalmente para análise e seleção de informações. Na Empresa B, somente as informações relativas a Vendas se encontram disponíveis no DW.
Os CI da Empresa A "concordam” que alterações foram feitas ou condicionadas com a opção de trabalhar com o DW. Na empresa B, os CI "discordam em parte" que alterações foram trazidas.

Nas empresas, a diferença de percepção em relação a alterações trazidas com o uso da ferramenta DW pode ser justificada pelo tempo de implementação, sendo também que na Empresa B a ferramenta DW se encontra implementada somente no setor de Vendas.

\subsection{Fase III - avaliação da qualidade da informação e do Data Warehouse}

Os itens analisados nesta fase são apresentados a seguir.

Dimensões da qualidade da informação - Em relação ao aspecto de como é a QI na perspectiva do CI nas empresas que utilizam DW, foram avaliadas 16 dimensões distribuídas em 4 categorias.

Na Empresa A, os CI "concordam" que existe qualidade na informação e, na empresa B, os consumidores "concordam em parte", tendo "discordado" na dimensão Integridade. Integridade, Completeza ou Perfeição é a extensão do quanto a informação não está extraviada e é suficiente para a tarefa em amplitude e profundidade. No DW, os problemas de Integridade podem ser causados por diversos motivos, tais como, carga incompleta na tabela de fatos e dimensões incompletas.

Outro aspecto importante é a forma como são tratados os valores nulos. Se existem problemas de avaliação da QI na perspectiva do CI, como no caso da Empresa B na dimensão Integridade, podem-se identificar suas possíveis causas. No caso, esta dimensão pertence à Categoria Contextual, em que os interesses da qualidade de dados e possíveis causas são respectivamente:

- Interesses da qualidade de dados: problemas operacionais da produção de dados, mudança de necessidade dos consumidores de dados e computação distribuída.

- Possíveis causas: dados incompletos, representação inconsistente, inadequação definida ou dados mensurados. Resultados de dados que não poderiam ser propriamente agregados.

É de interesse ressaltar que na visão ontológica descrita na literatura, a dimensão Integridade faz parte da visão interna (projeto, operação) de um SI que deve se preocupar com o uso e efeito desse sistema. Diz respeito ao propósito e justificações do sistema e seu desdobramento na organização e deve se preocupar com a construção e operação necessárias para atingir a funcionalidade requerida.

Nesse sentido, entende-se a avaliação da motivação para o uso da ferramenta na Empresa B e a "discordância" com a dimensão Integridade, uma vez que, atualmente, a 
ferramenta DW só está implementada no setor de Vendas e em processo em implantação nos demais setores.

Existência de qualidade de dados pobre - Nesta avaliação será verificada a existência de uma qualidade de dados pobre a fim de possibilitar fazer um cruzamento dos possíveis problemas que possam estar afetando a avaliação das dimensões e o grau de importância destas.

No caso da Empresa A, tem-se os seguintes itens analisados e a respectiva fase do processo a que pertencem:

- Difícil acesso à informação em um tempo razoável e informação não numérica é difícil de indexar - processo de armazenagem da informação; e

- Análise de conteúdo automatizada, em coleções de informações ainda não disponíveis; mudança da informação relevante e útil; fácil acesso que conflita com requisitos de segurança, privacidade e confidencialidade; e falta de fontes suficientes de computação - processo de utilização da informação.

$\mathrm{Na}$ Empresa B, tem-se:

- Múltiplas fontes da mesma informação, e a informação é produzida utilizando-se julgamentos subjetivos processo de produção da informação; e

- Mudança de informação relevante e útil - processo de utilização da informação.

Consumidores de Informação estão na fase do processo de utilização da informação, acessam e utilizam as informações para suas tarefas. A utilização pode envolver informações adicionais, agregação e integração. Neste caso específico, tem-se:

- Análise de conteúdo automatizada, em coleções de informação ainda não está disponível: se a informação tem relevância e algum valor agregado, o consumidor deve também ser capaz de agregar, manipular e utilizar essas informações para computar tendências.

- À medida que mudam as tarefas dos CI e o ambiente organizacional, a informação que é relevante e útil também muda: A informação somente é de alta qualidade se atender às necessidades das informações dos consumidores; somente eles fazem isso satisfazer o aspecto contextual da QI.

- Fácil acesso à informação pode conflitar com requisitos de segurança privacidade e confidencialidade. Para os CI, informação de alta qualidade é informação que é facilmente acessível. Garantir privacidade, confidencialidade e segurança da informação, entretanto, requer barreiras ao acesso. Neste caso, a acessibilidade e a segurança tendem ao conflito.

- Falta de fontes suficientes de computação limitam o acesso.

Grau de Importância das Dimensões e Categorias da QI - Será feita a formulação dos objetivos de cada dimensão para a seguinte questão: do ponto de vista do CI, quão importante é uma dimensão particular de qualidade comparada às outras dimensões no contexto da QI, solicitando a ordenação conforme o grau de importância do $1^{\circ}$ ao $5^{\circ}$. Esse procedimento será avaliado por meio da abordagem auto explicada, na qual os respondentes tacham os níveis de cada atributo de QI do mais para o menos preferido.

Dimensões mais importantes - Para os CI na Empresa A são: Relevância, Acuracidade, Credibilidade e Valor Agregado. Na empresa B são: Acuracidade, Reputação, Credibilidade e Interpretabilidade. Observa-se que Acuracidade e Credibilidade são dimensões importantes para ambas as empresas pesquisadas.

Conforme a literatura, "o senso comum aponta a Acuracidade como sendo a própria QI, sendo esta apenas uma das dimensões" (WANG; ZIAD; LEE, 2000) e sendo também para um ambiente coorporativo, no caso da Empresa $\mathrm{B}$, uma das mais importantes e relevantes dimensões da Categoria Intrínseca.

Categorias mais importantes - Os CI da Empresa A consideram a Categoria Contextual como sendo a mais importante, fato esse que, verificado na literatura, mostra nessa categoria a inserção da informação em seu meio de utilização. Essa categoria indica que não existem ou deveriam existir poucos problemas operacionais com a produção de dados. São observadas na literatura três causas básicas para que os consumidores de dados reclamem que os dados disponíveis não apoiam suas tarefas: falta de dados (incompletos), dados inadequadamente definidos ou medidos e dados que não puderam ser apropriadamente agregados.

Já na Empresa B, os CI deram maior importância à Categoria Intrínseca que, pela literatura, mostra o fato de a informação ter qualidade em si mesma. Diferenças entre fontes dos mesmos dados são causas comuns das preocupações. Inicialmente os consumidores de dados não sabem a fonte à qual problemas de qualidade devem ser atribuídos. Eles sabem somente que o dado é conflitante. Essa categoria representa a expectativa da inexistência de múltiplas referências de um mesmo dado e o julgamento envolvido na produção deste.

Avaliação do Data Warehouse - Apesar do interesse das empresas analisadas estarem coerentes com a Revisão da Literatura sobre as decisões que levaram à compra e adoção da ferramenta e quanto ao desenvolvimento do projeto ser elaborado conforme definição dos usuários nas empresas analisadas, não foi feito planejamento e atualmente não existe um gerenciamento focado na QI, não existindo assim um plano para a gestão, capacitação ou reciclagem nem a existência de procedimentos de avaliação. 
Numa visão geral, os CI da Empresa A "concordam" com a utilização da ferramenta DW. Para eles, existe a necessidade de capacitação para a utilização do DW, definição de "gerentes de projeto" que se responsabilizem por ele e não como respostas às demandas dos usuários. $\mathrm{O}$ volume de dados DW é grande, e, portanto, precisa-se aperfeiçoar a utilização desses dados por meio de equipes técnicas das diversas áreas, junto com a TI, necessitando-se de um "projeto" no sentido mais amplo, em que se crie uma metodologia eficiente de utilização dessas informações.

Na empresa B, os CI estão na escala "neutra", ou seja, nem concordam nem discordam quanto à utilização da ferramenta DW. Para eles o DW é uma boa ferramenta. Pelo processo estar ainda em fase de implantação, o escopo é limitado e o investimento ainda não se justificou em virtude da pouca utilização.

\section{Conclusões}

O presente estudo descreve e analisa como é a Qualidade da Informação gerada pelo Data Warehouse na perspectiva do Consumidor de Informação, do ponto de vista de vários autores, tendo, como objetivo principal, a análise da QI em duas empresas que utilizam o DW na perspectiva do CI e a verificação do grau de importância atribuída às dimensões e categorias da qualidade da informação, sendo, o sujeito do estudo, o Consumidor de Informação.

Por meio da aplicação do questionário, ficou claro o não conhecimento, por parte dos Consumidores de Informação, a respeito dos conceitos da ferramenta DW, da QI e dos métodos e técnicas estatísticas utilizadas para extração de informações.

A comparação dos resultados das avaliações da QI indica a importância atribuída pelos Consumidores de Informação às dimensões e categorias, levando-se em consideração que qualidade de dados é o "encontro consistente das "expectativas" dos Consumidores de Informação", e que um dado pronto para uso significa todas as dimensões atendendo o consumidor.

Pode-se, dessa forma, inferir que existe uma diferença entre o desejado e o real, fator que pode não estar atendendo à expectativa do Consumidor de Informação.

Numa manufatura de informação, o consumidor de um sistema de informação é parte do sistema e a matéria-prima utilizada no processamento da informação são os dados. Ela pode ser vista como um sistema agindo em dados brutos para produzir um produto de informação. Assim sendo, pode-se considerar que o produto final, ou seja, os dados processados no DW são Produtos de Informação.

Esta consideração baseia-se no simples fato de que o dado é tratado para ser aplicado pelo DW. Park (2006) verificou que, melhorando a qualidade de um SAD pela adição de um DW, pode-se melhorar a qualidade e disponibilidade da informação e, por consequência, melhorar o desempenho de decisão dos usuários do SAD.

Pelos resultados e análises do estudo, observa-se que o DW melhorou a qualidade da informação nas empresas avaliadas, porém é necessário mais treinamento em relação à disponibilidade de recursos e também do próprio conceito de "armazém de dados".

\title{
Information quality in Data Warehouse enterprises users
}

\begin{abstract}
Nowadays people are surrounded by a great deal of data that sometimes are redundant and not accurate. This data needs to be efficiently managed to be transformed into significant information for decision support. An enterprise can be more competitive if can obtain good quality information and can process this information quickly and securely. The use of a Data Warehouse tool enables the transformation of raw data into analytical information, but it requires data quality management. This article's main goal is to analyze perceived Information Quality (IQ) by Information Consumers in enterprises that use Data Warehouse. This analysis will be made using IQ dimensions and categories. A conceptual revision and interviews with users from two enterprises were made to reach the proposed objective. Users were divided into two categories. Aspects of IQ in these enterprises are presented.
\end{abstract}

Keywords: Information quality. Information system. Data Warehouse. Decision support. 
AL-HAKIM, L. Information quality function deployment. In: INTERNATIONAL CONFERENCE ON INFORMATION QUALITY (ICIQ 04), 9, 2004. Proceedings...

AMARAL, G. C. M.; CAMPOS, M. L. M. Modelando metadados de qualidade no data warehouse. In: SIMPÓSIO DE DESENVOLVIMENTO E MANUTENÇÃO DE SOFTWARE DA MARINHA, 4, 2004. Disponível em http://www.sdms.mar. mil.br/artigos/6918.pdf. 2004.

ANGELES, M. P. Detection and resolution of data inconsistencies, and data integration using information quality criteria. 2004. Disponível em: http://www.macs.hw.ac.uk:8080/techreps/docs/ files/HW-MACS-TR-0017.pdf

ARNDT, D.; LANGBEIN, N. Data quality in the context of customer segmentation. In: INTERNATIONAL CONFERENCE ON INFORMATION QUALITY (ICIQ-02), 7, 2002. Proceedings...

BURGESS, M. S. E.; GRAY, W. A.; FIDDIAN, N. J. Quality Measures and the Information Consumer. In: INTERNATIONAL CONFERENCE ON INFORMATION QUALITY, 9, 2004. Proceedings...

CASTELLS, M. A sociedade em rede a era da informação: Economia, Sociedade e Cultura. [S.1.]: Ed. Paz e Terra, 1999.

CHENOWET, T.; DOWLING, K. L.; ST LOUIS, R. D. Convincing DSS users that complex models are worth the effort. Decision Support Systems, v. 37, p. 71-82, 2004.

DOHERTY, N. F.; DOIG, G. An analisys of the anticipated cultural impacts of implemementation of data warehouse. IEEE Transactions on Engineering Management, v. 50, n. 1, Feb 2003.

FALSARELLA, O. M.; CHAVES, E. O. C. Sistemas de informação e sistemas de apoio à decisão. Revista de Informática, v. 3, n. 1, Jan-Jun 1995.

FAVARETTO, F.; MATTIODA, R. A. A. Medição da qualidade da informação: Um experimento na pesquisa em base de dados científicas. In: ENCONTRO NACIONAL DE ENGENHARIA DE PRODUÇÃO, 25, Nov. 2005, Porto Alegre.

INMON, W. H. Como construir o data warehouse. 2 ed. Rio de Janeiro: Campus, 1997.

LEE, Y. W. et al. AIMQ: A methodology for information quality assessment. Information and Management, v. 40, n. 2, p. 133-146, 2002 .
MATTIODA, R. A. A.; FAVARETTO, F. Qualidade de dados e produtos: Uma analogia. In: CONGRESSO NACIONAL DE EXCELÊNCIA EM GESTÃO - "SUSTENTABILIDADE NAS ORGANIZAÇÕES BRASILEIRAS: PÚBLICAS, PRIVADAS E DO TERCEIRO SETOR", 3, Ago. 2006, Niterói.

NOLAN, R. L. Management accounting and control of data processing. New York: National Association of Accountants, 1977.

PARK, Y. An empirical investigation of the effects of data warehousing on decision performance. Information \& Management, v. 43, p. 51-61, 2006.

PIPINO, L. L.; LEE, Y. W.; WANG, R. Y. Data Quality Assessment. Communications of the ACM, v. 45, n. 4, p. 211 - 218, April 2002.

POZZEBON, M.; FREITAS, H. M. R. Pela aplicabilidade - com maior rigor científico - dos estudos de caso em sistemas de informação. In: ENCONTRO DA ASSOCIAÇÃO NACIONAL DE PÓS-GRADUAÇÃO E PESQUISA EM ADMINISTRAÇÃO ANPAD, 21, 1997, Rio das Pedras.

SAKAGUCHI, T.; FROLICK, M. A. Review of the Data Warehousing Literature. Journal of Data Warehousing, v. 2, n. 1, p. 34-54, 1997.

SINGH, H. S. Data warehouse - conceitos, tecnologias, implementação e gerenciamento. São Paulo: Makron Books, 2001. p. 14.

STRONG, D. M.; LEE, Y. W.; WANG, R. Y. 10 Potholes in the Road to Information Quality. Computer, v. 30, n. 8, p. 38-46, Aug. 1997a.

STRONG, D. M.; LEE, Y. W.; WANG, R. Y. Data quality in context. Communications of the ACM, v. 40, n. 5, p. 103-110, May 1997b.

WAND, Y.; WANG, R. Y. Anchoring data quality dimensions in ontological foundations. Communications of the ACM, v. 39, n. 11, p. 86-95, 1996.

WANG, R. Y. A product perspective on total data quality management. Communications of the ACM, v. 41, n. 2, p. 58-65, 1998.

WANG, R. Y.; ZIAD, M.; LEE, Y. W. Data Quality. Hinghan, MA, USA: Kluwer Academic Publishers, 2000.

WANG, R. Y.; STRONG, D. M. Beyond accuracy: What data quality means to data consumers. Journal of management information Systems, v. 12, n. 4, Spring 1996.

\section{Sobre os autores}

\section{Rosana Adami Mattioda}

Programa de Pós-Graduação em Engenharia de Produção e Sistemas, Pontifícia Universidade Católica do Paraná Rua Imaculada Conceição, 1155, Curitiba - PR, CEP 80215-901

e-mail: mattioda@brturbo.com.br

\section{Fábio Favaretto}

Programa de Pós-Graduação em Engenharia de Produção e Sistemas, Pontifícia Universidade Católica do Paraná Rua Imaculada Conceição, 1155, Curitiba - PR, CEP 80215-901

e-mail: fabio.favaretto@ pucpr.br e professor.favaretto@gmail.com 